\title{
A IMPORTÂNCIA DE ARTEFATOS DO DESIGN DE INTERAÇÃO AO LONGO DO PROCESSO DE DESENVOLVIMENTO DE SOFTWARE: UM RELATO SOBRE A CRIAÇÃO DO APP COACH ZAC
}

\author{
THE IMPORTANCE OF INTERACTION DESIGN ARTIFACTS \\ THROUGHOUT THE SOFTWARE DEVELOPMENT PROCESS: \\ ACCOUNTING THE CREATION OS THE APP COACHZAC
}

\author{
Axel Junio Almeda de Andrade ${ }^{1}$, Bach. \\ Elton José da Silva², M.Sc. \\ (1) Universidade Federal de Ouro Preto \\ e-mail: axel.andrade@aluno.edu.ufop.br \\ (2) Universidade Federal de Ouro Preto \\ e-mail: elton@prof.edu.ufop.br
}

\begin{abstract}
Palavras-chave: design de interação, artefatos de design, aplicativos móveis.
O objetivo deste trabalho é apresentar o processo de design de interação de um protótipo de um aplicativo de mobile learning para interessados no jogo de tênis, focado na avaliação do saque, um dos fundamentos do esporte mais difíceis de dominar. $O$ aplicativo será uma ferramenta para auxiliar o treinador na análise do movimento corporal do saque, trazendo benefícios para o treinamento dos atletas. O relato sobre o processo de design e os diversos artefatos gerados ao longo do mesmo pode ser de interesse tanto de acadêmicos quanto profissionais da área de desenvolvimento de aplicativos móveis.
\end{abstract}

Key-words: interaction design, design artifacts, mobile applications.

The purpose of this work is to present the interaction design process of a mobile learning application prototype for those interested in the game of tennis, focused in the service evaluation, one of the most difficult fundamentals of this sport. The app aims to be a tool to assist the trainer in the analysis of the corporal movement at serving, bringing benefits to the training of the athletes. The accounting of the design process and the artifacts generated throughout it, may be of interest to both academics and professionals in the field of mobile application development.

\section{Introdução}

A obtenção de sistemas interativos com boas interfaces depende da habilidade da equipe entender e avaliar - e consequentemente melhorar - o projeto durante $\mathrm{o}$ processo de desenvolvimento. Este entendimento depende, em sua maior parte, dos artefatos gerados para representação do design durante $\mathrm{o}$ processo de desenvolvimento. A necessidade de artefatos efetivos é fundamental no desenvolvimento que enfatiza refinamentos sucessivos e envolve diferentes grupos de trabalho para a produção de um sistema [Hix, 1993].

O objetivo deste trabalho é apresentar o processo de design de interação de um protótipo de um aplicativo de mobile learning para interessados no jogo de tênis, focado na avaliação do saque que é considerado o golpe mais importante do tênis. Com um bom saque o jogador pode ditar o ritmo do jogo, tendo todo controle do ponto [Ishizaki, 2006]. O aplicativo será uma ferramenta para auxiliar o treinador na análise do movimento corporal do saque, trazendo benefícios para o treinamento dos atletas. É crescente o interesse pela área de mobile learning, onde os smartphones e demais dispositivos tecnológicos que se conectam com a internet, são responsáveis por auxiliar no ensino e na aprendizagem, criando novos ambientes de aprendizagem à distância, permitindo atualizações mais rápidas de conteúdos para reduzir o tempo de aprendizado [Marçal et al., 2009] [Mülbert, 2011]. 


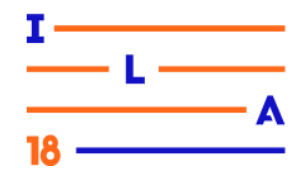

O relato apresentado ao longo do artigo sobre o processo de design e os diversos artefatos gerados ao longo do mesmo, como requisitos, modelo conceitual, metáforas, personas, modelos de tarefas, interação e dados, e protótipos de baixa e alta fidelidade, pode ser de interesse tanto de acadêmicos quanto profissionais da área de desenvolvimento de aplicativos para aplicações móveis.

O restante deste artigo está organizado da seguinte forma; na seção 2 é apresentada a idéia do aplicativo CoachZac, comparando-o com alguns aplicativos disponíveis no mercado. $\mathrm{Na}$ seção 3, é apresentada uma visão geral do processo de design de interação utilizado na construção do aplicativo. $\mathrm{Na}$ seção 4, são detalhados os artefatos que foram gerados ao longo do processo de design. Na seção 5 é apresentada uma discussão dos resultados. Finalmente, na seção 6 são apresentadas as conclusões e sugestões de trabalhos futuros.

\section{O Aplicativo CoachZac}

Neste artigo é apresentado o processo de design do aplicativo CoachZac, de mobile learning, que visa dar apoio a treinadores e atletas na avaliação dos movimentos corporais do saque no tênis. O saque é um dos fundamentos mais importantes deste esporte, sua boa execução é a oportunidade de ter o domínio sobre o ponto, obrigando o adversário a jogar em defensiva. A realização do fundamento do saque é de difícil domínio, uma vez que vários fatores podem afetá-lo. Por exemplo, em relação à altura que a bola deve ser lançada, é recomendável que ela atinja o ponto onde a soma da extensão do braço e da raquete seja máxima. A bola não deve ser elevada acima desse ponto, para que o jogador não seja obrigado a esperar a bola cair para atingi-la. Por outro lado, se a bola for elevada muito abaixo do ponto correto, a raquete ainda não estará na altura correta para a batida [Treuherz, 2006].

Atualmente, já existem diversos aplicativos, com foco no treino dos fundamentos do tênis, disponíveis no mercado. Como trabalhos relacionados, foram escolhidos dois dos principais aplicativos encontrados na Google Play, com a justificativa de que os seus objetivos são semelhantes aos do CoachZac. O Tennis Training [Fitivity, 2018], por exemplo, é um aplicativo gratuito, desenvolvido pela empresa Fitivity, especializada desenvolver aplicativos de aprendizado de diversos esportes. De acordo com o autor o objetivo é ensinar a executar diferentes tipos de técnicas e ao mesmo tempo proporcionar exercícios para prática dos fundamentos do tênis.

As principais funcionalidades do aplicativo são: orientação por áudio, personalização de exercícios semanais, vídeos em alta definição para cada treino e fluxo exercícios on-line e offline. A Figura 1a apresenta a tela de personalização dos exercícios que devem ser treinados naquela semana. Ao selecionar um fundamento, é possível visualizar uma série de vídeos com todos os passos para uma boa execução. A Figura $1 b$ apresenta a personalização do treino, onde os vídeos são executados, podendo controlar o número de repetições.

Como ponto positivo destaca-se o acervo de vídeos contendo diversos fundamentos $\mathrm{e}$ demonstrando a maneira correta de serem executados, porém, a principal diferença para o CoachZac é que não há nenhuma funcionalidade no aplicativo que dê um feedback, no que diz respeito a execução dos fundamentos, para os atletas. 
Interaction Latin America

Rio de Janeiro, Brasil - 2018

http://ila.ixda.org/2018

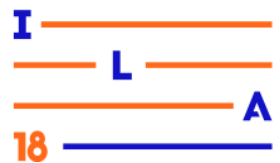

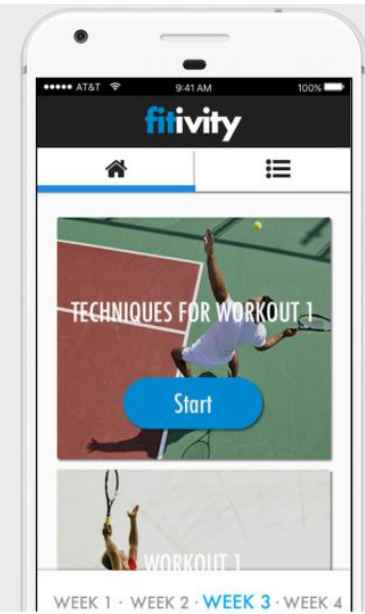

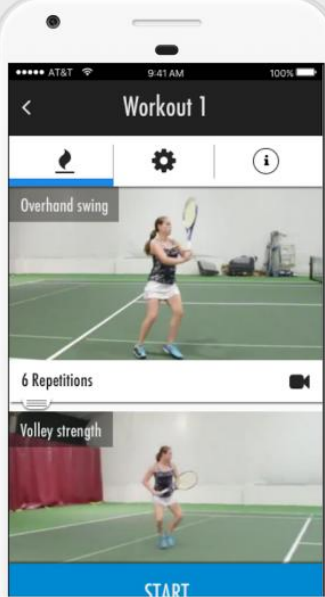

Figura 1: TennisTraining (a) Personalização semanal (b) Personalização do treino

O Tennis Coach Plus [Zappasoft, 2018], por sua vez, é um aplicativo pago, que tem o objetivo permitir ao treinador gerar um feedback visual, através da análise de vídeos. Foi desenvolvido pela empresa Zappasoft, que tem diversos aplicativos, nesta mesma linha para outros esportes. É possível analisar vários fundamentos do tênis, incluindo o saque. $\mathrm{O}$ aplicativo possui funcionalidades como a importação de vídeos da biblioteca, análise através da reprodução do vídeo, reprodução de dois vídeos ao mesmo tempo lado a lado, e também permite a sobreposição desses vídeos. A Figura 2 apresenta os fundamentos que podem ser selecionados para avaliação e as funcionalidades citadas.

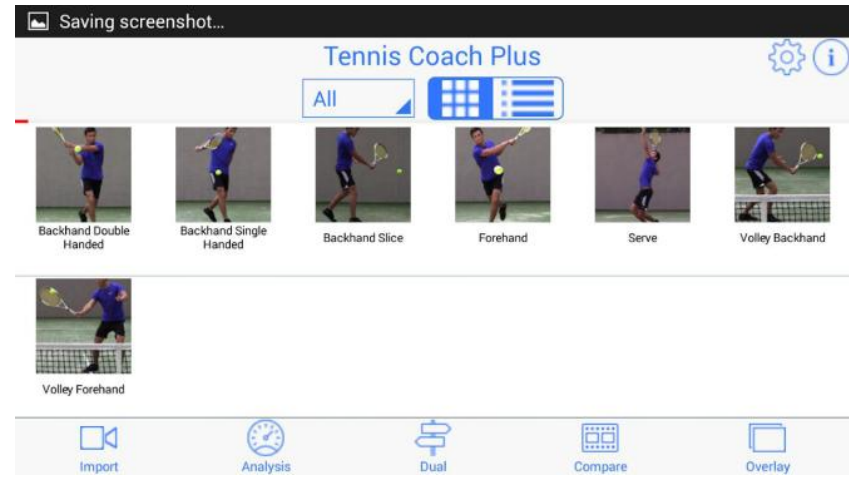

Figura 2 - Tennis Coach Plus, seleção de fundamentos

No momento da análise do vídeo, existem outras funcionalidades que merecem ser destacadas, como, anotações de texto ou de voz, zoom e velocidade de reprodução. A Figura 3 apresenta a avaliação de dois vídeos em paralelo e as funcionalidades que podem ser utilizadas pelo avaliador.

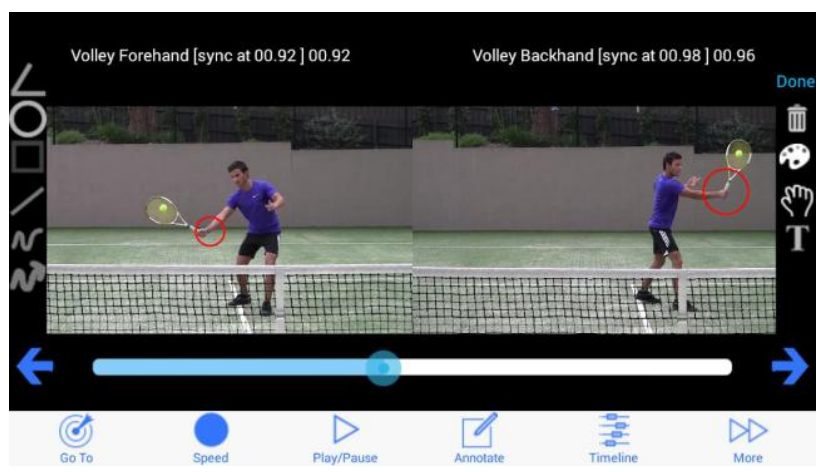

Figura 3 - TennisCoach Plus, avaliação de vídeos em paralelo.

O Tennis Coach Plus propõe um fluxo de interação bastante parecido com o CoachZac aqui proposto e ambos os projetos têm como usuários em potencial treinadores e atletas de tênis. Uma das vantagens do Tennis Coach Plus é a possibilidade de analisar diversos fundamentos do tênis, e também, a ideia de usar outros vídeos como base para análise.

A principal diferença em relação ao CoachZac está na forma em que os dados são coletados e nos resultados gerados por ele. No Tennis Coach Plus, o treinador, mesmo com o auxílio do vídeo, ainda necessita realizar anotações ou lembretes de forma manual, e os resultados são gerados através de vídeos com as edições feitas pelo treinador e também em pdf com screenshots das telas. O objetivo do CoachZac é facilitar ainda mais este processo para o treinador, melhorando a interação com o aplicativo e permitindo a produção de estatísticas inteligentes, que possibilitem a melhoria no desempenho do atleta.

\section{Processo de Design de Interação}

Para alcançar o produto final foi utilizada uma variação do modelo do Ciclo de Vida Simples para IHC [Preece et al., 2007], que pode ser dividido em quatro etapas principais: (i) identificação dos requisitos, (ii) design ou redesign, (iii) construção de uma versão interativa e (iv) avaliação, como ilustra a Figura 4. Esse modelo de processo é bastante iterativo e as etapas podem ocorrer repetidas vezes, limitada pelo orçamento, tempo e recursos disponíveis [Barbosa, 2010]. 
Interaction Latin America

Rio de Janeiro, Brasil - 2018

http://ila.ixda.org/2018

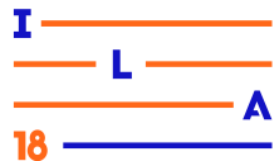

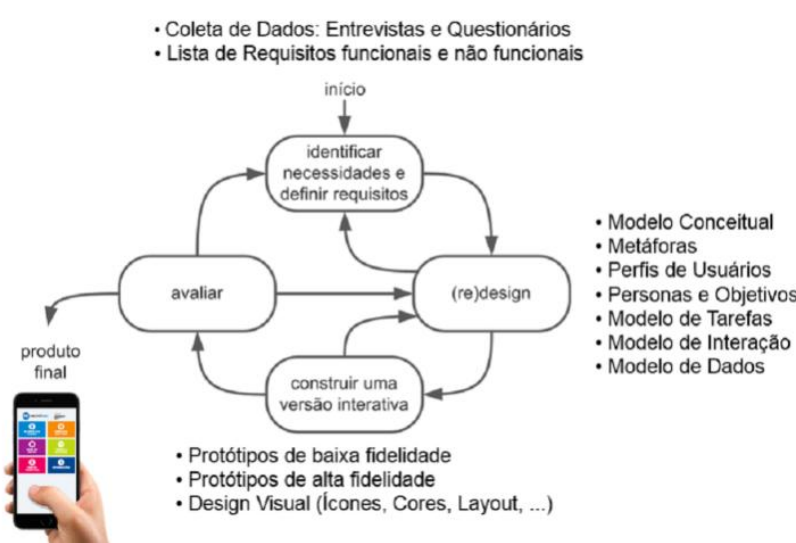

Figura 4 - Processo de Design com Artefatos Gerados

$\mathrm{Na}$ etapa inicial, o designer faz uma análise de necessidades do produto a ser construído e coleta, por exemplo, através de observações, entrevistas e questionários, os requisitos tanto funcionais quanto não funcionais. Na etapa de (re)design, uma das mais criativas do ciclo de vida, o designer elabora diferentes artefatos para representar as diversas ideias e alternativas que tem em mente, como por exemplo: perfis de usuários, personas e objetivos associados, modelo de tarefas, modelo de interação, modelo de dados, projeto visual da interface, incluindo projeto de icones, fontes, mensagens, layout e cores. É nesta etapa também que são gerados protótipos que podem ir das representações em papel até representações de alta fidelidade utilizando ferramentas computacionais. Nos protótipos, o designer também reflete sobre os diversos estilos de interação, com sua aparência e comportamento na interface. O processo de design geralmente é concluído com uma avaliação da solução proposta com métodos como avaliação heurística, inspeção semiótica e método de avaliação de comunicabilidade [Preece et al., 2007].

Um dos diferenciais desse processo é o envolvimento dos usuários no processo de construção do software desde as etapas iniciais, para aprender sobre as suas necessidades e assim influenciar positivamente na solução, bem como identificar e corrigir novos problemas aplicando o processo de design novamente quando necessário.

\section{Desenvolvimento do CoachZac}

Nesta seção são descritos os artefatos gerados durante o processo de desenvolvimento do protótipo do CoachZac, que inclui a lista de requisitos, o modelo conceitual, a metáfora da aplicação, as personas e objetivos com cenários de interação, o modelo hierárquico de tarefas, o fluxo de interação e protótipos.

O ciclo de vida teve início com entrevistas e questionários com stakeholders para entender melhor o problema e identificar as necessidades dos usuários. Foram realizadas reuniões com técnico e jogadores de tênis com objetivo levantar requisitos funcionais e não funcionais do sistema, coletar informações sobre como é feita a avaliação do fundamento do saque, identificar as dificuldades e também discutir possíveis soluções para melhorar o engajamento dos atletas nos treinos através do uso do aplicativo. Um questionário disponibilizado no Google Forms, foi submetido a dois grupos de jogadores no WhatsApp, com objetivo de identificar possíveis perfis, construir personas e também confirmar o problema enfrentado pelos jogadores na execução do fundamento do saque. Foram obtidas 76 respostas.

Dentre os resultados obtidos podemos destacar, por exemplo, que $78.9 \%$ dos jogadores responderam que praticam o tênis há 4 anos ou mais, $10.5 \%$ há 2 anos, $3.9 \%$ há 3 anos, 3.9\% há 1 ano e $2.6 \%$ menos de 1 ano. Em relação à prática do esporte, $65.8 \%$ como atividade física, $25 \%$ por passatempo e $9.2 \%$ com objetivo de ser um atleta profissional. Quando perguntados sobre como qualificam os seus saques, numa escala de 1, para péssimo e 10, para excelente, $19.7 \%$ nível $5,19.7 \%$ nível $8,18.4 \%$ nível 7 , $14.5 \%$ nível $6,13.2 \%$ nível $4,6.6 \%$ nível 5, $5.3 \%$ nível 9 e $2.6 \%$ nível 1.0 s resultados obtidos mostram que embora $78.9 \%$ dos jogadores pratiquem o tênis há 4 anos ou mais, o número de jogadores com nível do saque entre 1 e 6 é de $56.6 \%$, ou seja, mesmo praticando o esporte há muito tempo, a maioria dos jogadores não possuem um saque com nível considerado acima da média.

\subsection{Lista de Requisitos}

Os requisitos de um sistema são as descrições do que o sistema deve fazer, os serviços que oferece e 
as restrições a seu funcionamento. Esses requisitos refletem as necessidades dos clientes para um sistema que serve a uma finalidade determinada, como controlar um dispositivo, colocar um pedido ou encontrar informações [Sommerville, 2011]. Existem dois tipos de classificação para os requisitos, que são: requisitos funcionais e nãofuncionais. Os requisitos funcionais são declarações de serviços que o sistema deve fornecer, de como o sistema deve reagir a entradas específicas e de como o sistema dever se comportar em determinadas situações. Em alguns casos os requisitos funcionais também podem explicitar o que o sistema não deve fazer [Sommerville, 2011]. Os requisitos não funcionais referem-se critérios que qualificam os requisitos funcionais, como: desempenho, usabilidade, confiabilidade, segurança e etc.

A partir da análise de outros aplicativos apresentados na seção 2 e das reuniões feitas com stakeholders foram levantados os requisitos funcionais para o CoachZac, exemplificados na Tabela 1 a seguir:

\begin{tabular}{|c|c|}
\hline RF1 & $\begin{array}{l}O \text { aplicativo deve ter duas opções de entrada, uma } \\
\text { pelo facebook e outra por login e senha. }\end{array}$ \\
\hline RF2 & $\begin{array}{l}\text { O aplicativo deve permitir ao atleta visualizar somente } \\
\text { o resultado de seus treinamentos. }\end{array}$ \\
\hline RF3 & $\begin{array}{l}\text { O aplicativo deve permitir ao treinador visualizar } \\
\text { todos os resultados de qualquer atleta. }\end{array}$ \\
\hline RF4 & $\begin{array}{l}\text { O aplicativo deve permitir ao treinador realizar } \\
\text { avaliações de diferentes atletas. }\end{array}$ \\
\hline RF5 & $\begin{array}{l}\text { O aplicativo deve permitir ao treinador realizar a troca } \\
\text { do atleta a qualquer momento. }\end{array}$ \\
\hline RF6 & $\begin{array}{l}\text { O aplicativo deve permitir aos usuários a edição de } \\
\text { seus dados a qualquer momento. }\end{array}$ \\
\hline RF7 & $\begin{array}{l}\text { O aplicativo deve permitir gravar um vídeo ou abrir } \\
\text { um a partir da galeria, para iniciar o fluxo de } \\
\text { avaliação do saque. }\end{array}$ \\
\hline RF8 & $\begin{array}{l}\text { O aplicativo deve permitir ao treinador gravar áudios } \\
\text { e escrever anotações durante a avaliação. }\end{array}$ \\
\hline RF9 & $\begin{array}{l}\text { Para cada passo do fundamento saque, o aplicativo } \\
\text { deve apresentar um marcador, que será avaliado } \\
\text { pelo treinador de forma qualitativa. }\end{array}$ \\
\hline
\end{tabular}

Tabela 1 - Lista de Requisitos Funcionais

A Tabela 2 apresenta exemplos de requisitos não funcionais.

\begin{tabular}{|l|l|}
\hline RNF1 & $\begin{array}{l}\text { A interface do sistema deverá ser responsiva, ou } \\
\text { seja, se comporta adequadamente independente } \\
\text { do front-end que será utilizado para acesso - } \\
\text { Smartphone ou Tablet. }\end{array}$ \\
\hline RNF2 & $\begin{array}{l}\text { Todos os registros de atletas cadastrados no } \\
\text { sistema poderão ser mantidos } \\
\text { (alterados/consultados/excluídos) com o sistema } \\
\text { off-line e novos registros poderão ser incluídos } \\
\text { também com o sistema off-line }\end{array}$ \\
\hline RNF3 & $\begin{array}{l}\text { Ao registrar uma avaliação, os resultados devem } \\
\text { aparecer em, no máximo, 4 segundos }\end{array}$ \\
\hline
\end{tabular}

\begin{tabular}{|l|l|}
\hline RNF4 & $\begin{array}{l}\text { Os resultados devem ser compartilhados com os } \\
\text { atletas em um documento no formato pdf. }\end{array}$ \\
\hline RNF5 & $\begin{array}{l}\text { Testes Unitários e de Aceitação deverão ser } \\
\text { completamente automatizados }\end{array}$ \\
\hline
\end{tabular}

Tabela 2 - Lista de Requisitos Não Funcionais

\subsection{Modelo Conceitual}

O modelo conceitual é um conjunto de suposições baseadas no mundo real que indicarão as regras de negócio de um sistema. Esta etapa independe da escolha de tecnologias e protótipos ajudam no entendimento dos processos e tem como objetivo que o sistema possa ser compreendido pelo usuário da maneira pretendida pelo designer. Um modelo conceitual pode ser obtido pelas respostas a perguntas como $O$ que o sistema deve fazer? Como ele deve se comportar? Como ele deve se parecer?

De acordo com [Preece et al., 2007] modelos conceituais podem ser classificados em dois grupos, os modelos conceituais baseados em objetos, onde as interfaces remetem a objetos comuns do cotidiano, e em modelos conceituais baseados em atividades. $\mathrm{O}$ modelo conceitual proposto para o CoachZac possui uma abordagem híbrida combinando elementos dos modelos conceituais baseados em objetos, utilizando figuras e metáforas da modalidade de tênis e também dos modelos baseados em atividades, onde todo o fluxo da aplicação é dado por manipulação e navegação (uma classificação de modelo conceitual baseado em atividades).

\subsection{Metáforas de Interface}

As metáforas existem para facilitar a explicação e/ou compreensão de algo que está sendo contado ou apresentado. As metáforas de interface são baseadas em modelos conceituais que combinam conhecimento familiar e novos conceitos. Um mecanismo familiar direciona um usuário a entender, aprender e utilizar um novo produto ou equipamento [Barbosa, 2010].

As metáforas propostas para o CoachZac envolvem $\mathrm{o}$ treinador, Zac, avaliando $\mathrm{o}$ movimento corporal do saque dos jogadores através de uma prancheta com formulários estatísticos e evolução do treinamento. A idéia é utilizar na interface metáforas do tênis e que personalizem o papel do treinador, numa relação de apoio e orientação no processo de 


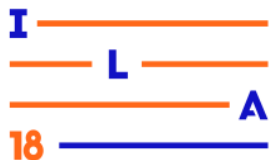

feedback nos treinamentos de saque dados aos atletas. A figura 5 a seguir apresenta exemplos de metáforas do aplicativo CoachZac.
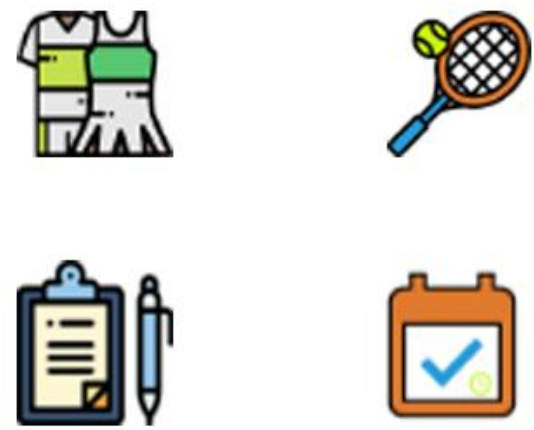

Figura 5 - Exemplos de metáforas no CoachZac

\subsection{Personas\&Objetivos}

A persona é um personagem fictício que representa um grupo de usuários reunindo padrões de comportamento acerca de suas decisões de compra, uso de tecnologia ou determinado produto, como descrito por [Barbosa, 2010]. Uma persona é definida por seus objetivos pessoais, práticos e orientados para a empresa, bem como pelo relacionamento com o produto a ser projetado, as emoções da persona ao usar o produto e os objetivos da persona em usar o produto. Em outras palavras, são os objetivos dos usuários que são o foco das descrições de persona, por exemplo, fluxo de trabalho, contextos e atitudes [Soegaard, 2013]. A elaboração de uma persona, tipicamente, inclui detalhes como: nome, idade, sexo, ocupação, expectativas e outros detalhes relevantes ao produto.

As fotos utilizadas na criação das personas são imagens livres retiradas do Google, sem restrições de uso, compartilhamento ou modificações. Para o CoachZac foram criadas quatro personas. Duas delas são ilustradas nas figuras 6 e 7 a seguir:
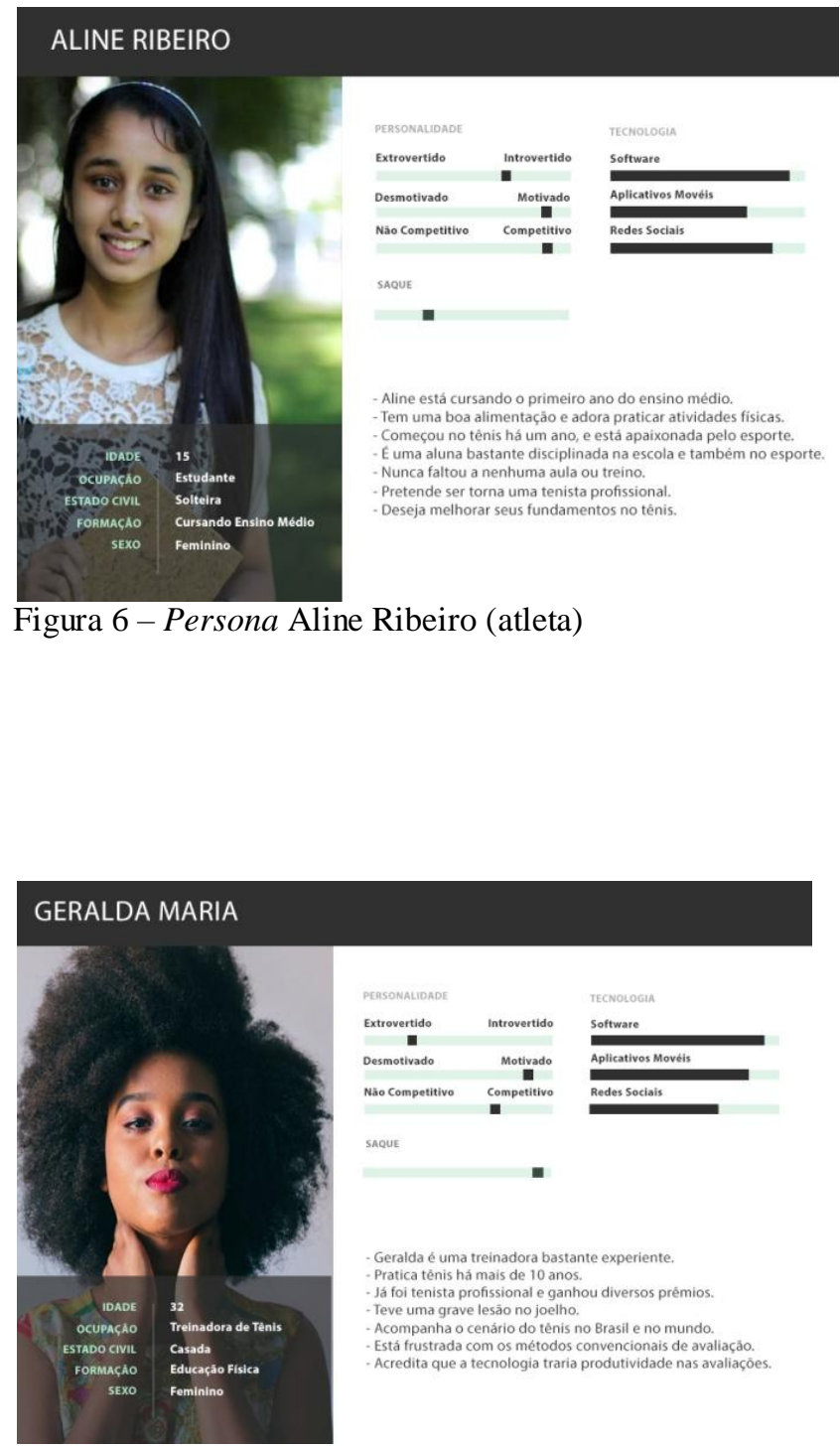

Figura 7 - Persona Geralda Maria (coach)

\subsection{Cenário de Interação}

Um cenário é "simplesmente uma história sobre pessoas executando uma atividade" [Rosson , 2002]. Os cenários ajudam a comunicar a essência da idéia de produto/serviço dentro de um provável contexto de uso e tem influência direta na forma de se pensar no design de interação e na solução.

Como os cenários são geralmente escritos em linguagem natural, o seu uso motiva todos os interessados no sistema a participarem e contribuírem com as decisões de design, direta ou indiretamente [Barbosa, 2010]. 


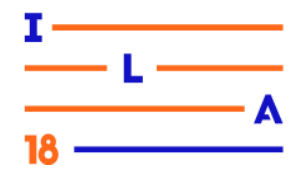

A seguir um exemplo de cenário de interação para avaliação do saque com os atores: Aline (atleta) e Geralda (treinadora).

"Nos primeiros treinos de Aline, Geralda percebeu que a jovem tinha um grande potencial. Para a atleta desenvolver o nível de seu saque, Geralda intensificou os treinos $e$ avaliações, que eram feitas somente aos sábados no clube. Aline começou a treinar em casa, gravando vídeos do movimento corporal de seus saques e enviando para que Geralda avaliasse. Para avaliá-los, Geralda deve fazer o download dos vídeos e utilizar o CoachZac. $O$ CoachZac, deve informar a Geralda, no momento de abertura de um vídeo, se o arquivo selecionado está no formato correto ou está corrompido. Caso não haja nenhum problema, Geralda deve realizar a avaliação do saque, e o CoachZac, deve unir os resultados da avaliação atual com os demais resultados de Aline, gerando um arquivo em pdf, contendo os resultados da atleta. Esses resultados devem ser enviados para Aline, antes do próximo treino. Geralda deve ser ágil na troca de feedbacks com Aline, para que atleta não realize treinos desnecessários, e tenha foco nos passos que está executando de forma incorreta. Ao receber os resultados, Aline deve identificar seus erros e realizar treinos para corrigi-los. "

\subsection{Diagrama Hierárquico de Tarefas}

Um modelo de tarefas é uma descrição hierárquica das atividades a serem executadas para alcançar os objetivos do usuário. A modelagem de tarefas deste trabalho foi baseada na Análise Hierárquica de Tarefas, proposto por [Preece et al., 2007], que divide as tarefas em planos de ação.

Para a análise hierárquica do modelo de tarefas do CoachZac, foram considerados dois fluxos, um para o treinador e outro para o jogador. A Figura 8 apresenta o resultado final da análise hierárquica de tarefas para o treinador, onde fluxo encontrado é praticamente horizontal, como mostra o plano de ação 0-1-2-3-4-5. Existem outros fluxos secundários como 0-1-23-3.1 e o 0-1-2-3-4-4.1.

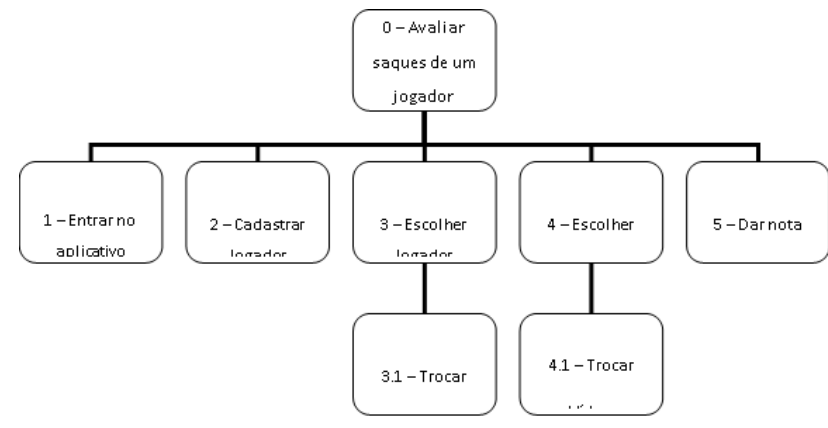

Figura 8-Diagrama de Tarefas para o CoachZac

\subsection{Modelo de Interação}

Uma modelagem de interação ou diagrama de interação do usuário representa o fluxo de interação entre o usuário e uma aplicação com intensa troca de informações e suporte à navegação. A MoLIC(Modeling Language for Interaction as Conversation) foi projetada para apoiar os designers no planejamento da interação, motivando sua reflexão sobre as estratégias de realização de atividades e resolução de problemas dos usuários que deveriam ser apoiadas pelo sistema interativo [Barbosa, 2010]. O diagrama de interação representa como os objetivos poderão ser atingidos durante a interação. Assim como cenários e modelos de tarefas, o diagrama de interação MoLIC serve como ponte entre a definição dos objetivos dos usuários e o projeto da interface propriamente dita [Barbosa, 2010].

A Figura 9 a seguir representa o modelo de interação proposto para o login no sistema, escolha do atleta, escolha do vídeo e avaliação do saque.

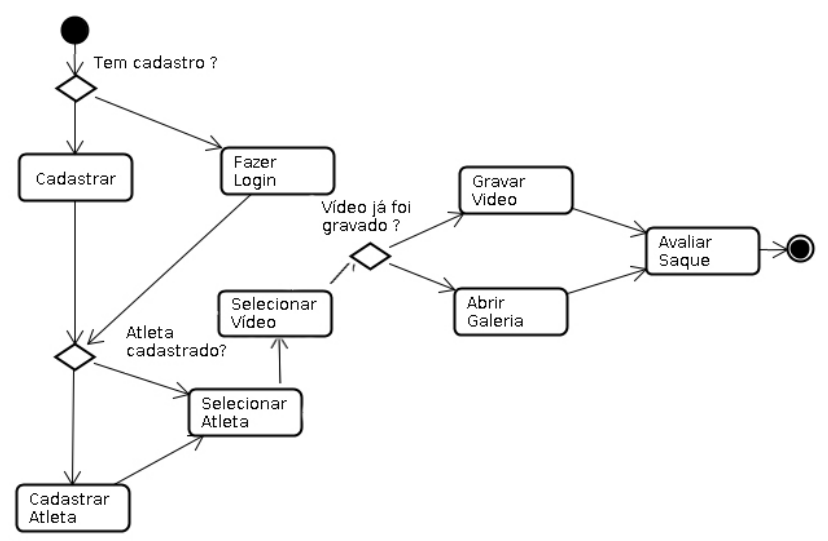

Figura 9 - Diagrama de Interação para o CoachZac 


\subsection{Modelo de Dados}

A modelagem conceitual é uma fase muito importante no planejamento de uma aplicação de um banco de dados bem-sucedida. O Modelo Entidade-Relacionamento (ER) é um modelo de dados conceitual de alto nível, além de muito popular. Esse modelo e suas variações são normalmente empregados para $\mathrm{o}$ projeto conceitual de aplicações de um banco de dados, e muitas ferramentas de projeto de um banco de dados aplicam seus conceitos [Elmasri, 2011].

A Figura 10 a seguir apresenta o modelo de entidades e relacionamentos estendido desenvolvido para o CoachZac, com as entidades, atributos e relacionamentos.

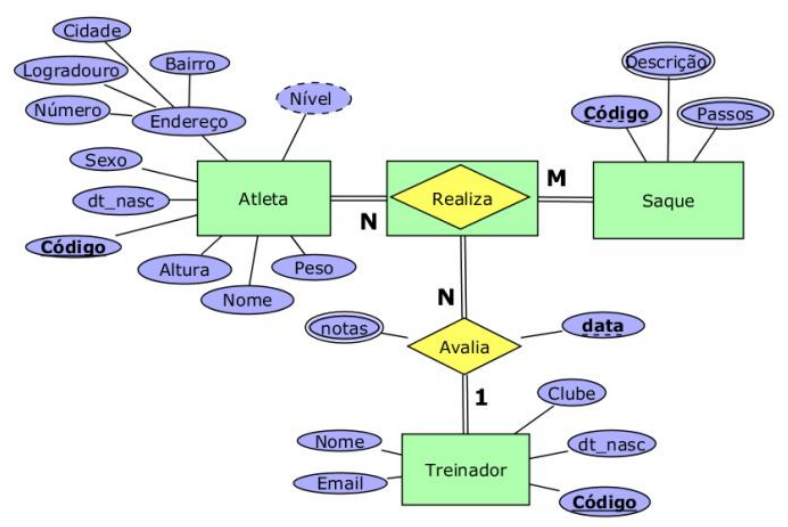

Figura 10 -Modelo ER para o CoachZac

\subsection{Protótipos}

A prototipação no desenvolvimento de software é um processo que tem como objetivo avaliar as idéias geradas e validar ou não todos os requisitos estabelecidos. Essa etapa é importante para verificar se a solução desenhada está adequada ao desafio que o cliente enfrenta, garantindo o alinhamento das informações, melhorando a comunicação dos desenvolvedores com os usuários, identificando de forma ágil os requisitos que não foram aplicados, analisando a experiência do usuário com o software e reduzindo o número de remodelagens no desenvolvimento do projeto.
Nas seções 4.9.1 e 4.9.2, são apresentadas as fases da prototipação de baixa e alta fidelidade, respectivamente.

\subsubsection{Protótipo de baixa fidelidade}

Os protótipos de baixa fidelidade são representações conceituais, ou seja, não necessariamente se assemelham ao produto final. Também são conhecidos como rascunhos, wireframes ou sketches. A Figura 10 a seguir ilustra algumas telas do protótipo de baixa fidelidade para o CoachZac. Foi utilizada a ferramenta Photoshop para a construção desses protótipos. A Figuras 11 a seguir apresenta algumas telas do protótipo de baixa fidelidade criado para o aplicativo CoachZac:
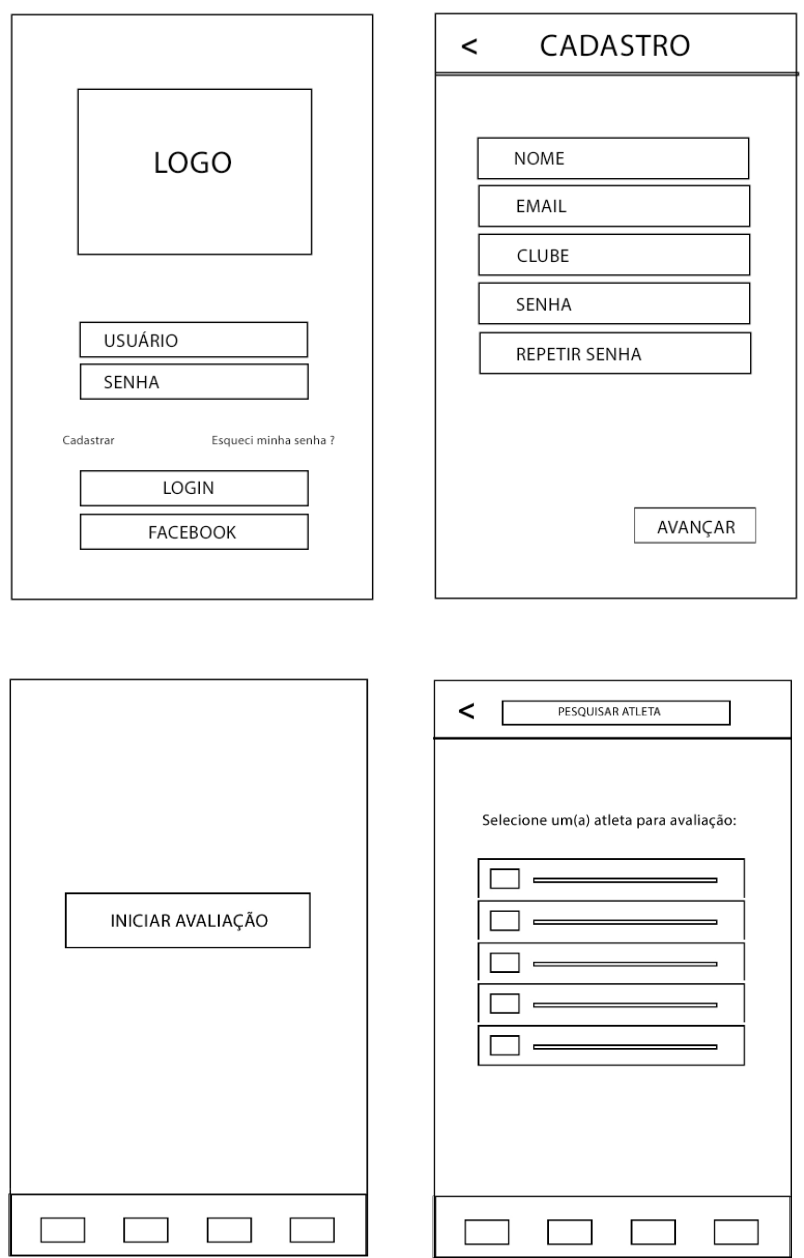

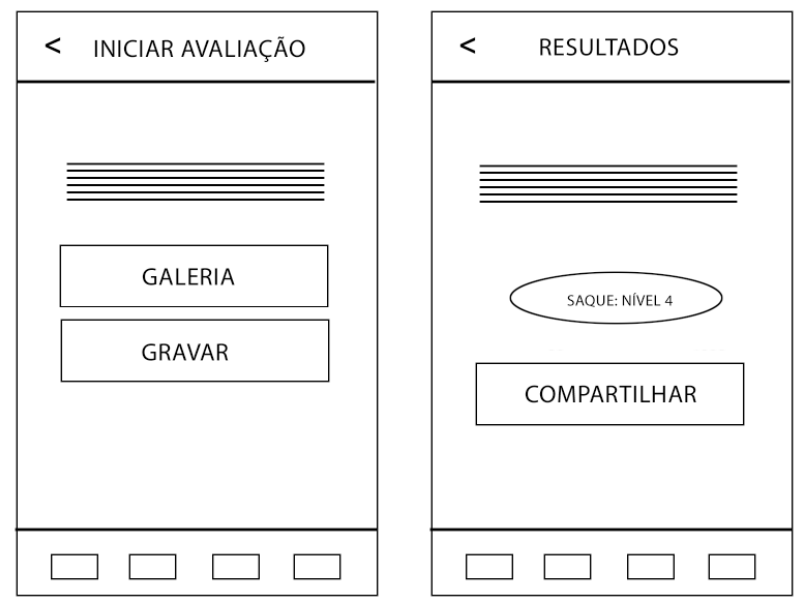

Figura 11 - Protótipos de baixa fidelidade

\subsubsection{Protótipos de alta fidelidade}

Os protótipos de alta fidelidade utilizam ferramentas computacionais e permitem simular o comportamento de interação e da interface de um sistema sendo construído. Esta fidelização é considerada um mock-up, de forma que seja o mais próximo possível do resultado final do software. Este tipo de protótipo demanda maior tempo para ser produzido, porém com um resultado mais eficiente. Sendo o mock-up uma simulação do que será o software, os usuários podem validar todas as funcionalidades em tempo real, já também com uma representação visual do mesmo.

O desenvolvimento nesta etapa normalmente é realizado na finalização da interface, já com programas de design gráfico, ferramentas de codificação front-end e linguagens de programação. Neste trabalho foi escolhida a ferramenta Adobe Xd para construção do protótipo de alta fidelidade. A escolha se justifica pela facilidade na criação e na conexão das telas e também a possibilidade de exportação do projeto para $w e b$, facilitando assim os feedbacks de interação e usabilidade a partir de testes com usuários.

As Figuras 12 a 17 apresentam algumas telas do protótipo de alta fidelidade criado para o aplicativo CoachZac. A figura 12a apresenta a tela de login para o treinador, onde é possível se logar com usuário e senha e também pelo facebook. A figura $12 \mathrm{~b}$ apresenta a tela para recuperar senha, é necessário informar o email do usuário para realizar o processo de recuperação.
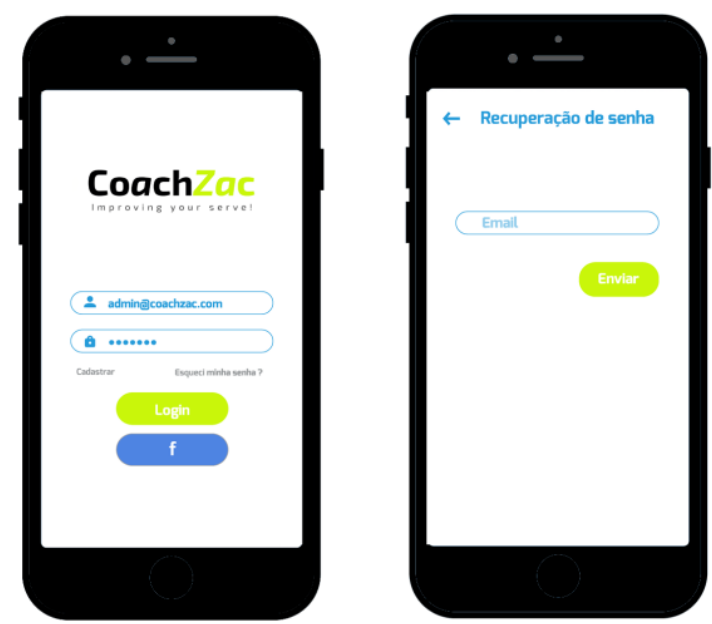

Figura 12 -(a) Login (b) Recuperação de senha

A figura 13a apresenta a tela de cadastro para o treinador com os seguintes campos: nome, email, clube, senha e repetir senha. A figura $13 \mathrm{~b}$ apresenta a tela inicial, é possível iniciar uma avaliação.
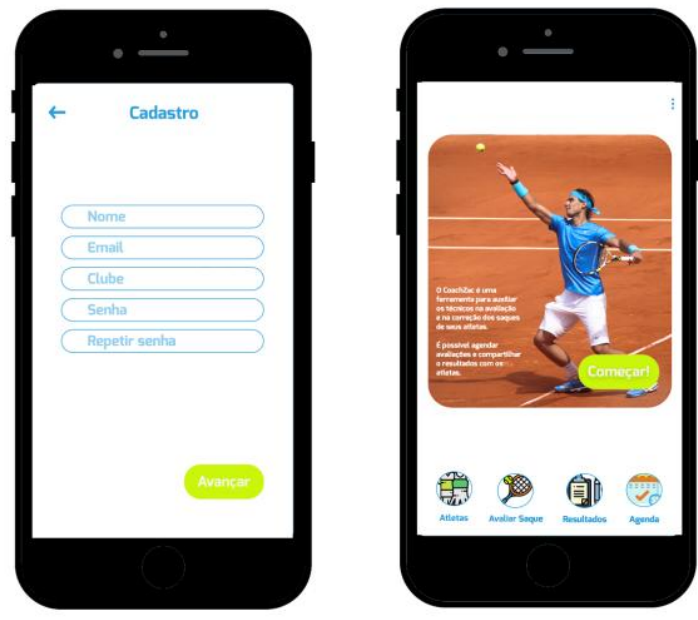

Figura 13 - (a) Cadastro treinador (b) Iniciar avaliação

A figura 14a apresenta a tela de gerenciamento dos atletas, o treinador pode visualizar os perfis, encontrar atletas pelo nome, adicionar atletas, remover atletas, filtrar a busca e iniciar uma avaliação. A figura 14b apresenta a tela de perfil do atleta, o treinador visualiza todas as informações do atleta, e consegue ver os resultados e inicializar uma avaliação desse atleta. 
Interaction Latin America

Rio de Janeiro, Brasil - 2018

http://ila.ixda.org/2018

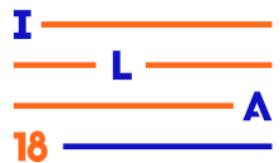

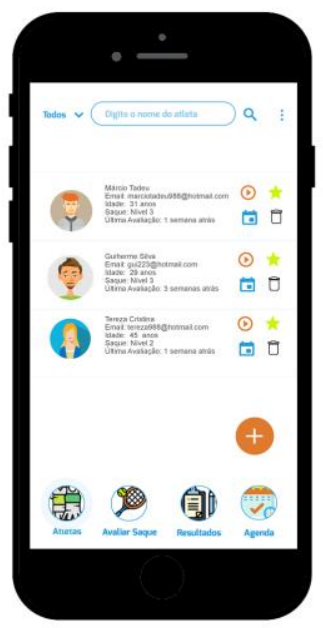

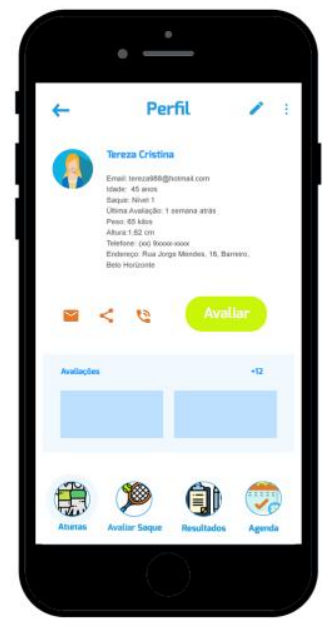

Figura 14 - (a) Gerenciamento de atletas (b) Perfis

A Figura 15a apresenta a tela de seleção de atletas para avaliação. A figura $15 \mathrm{~b}$ apresenta a tela de seleção do vídeo que será avaliado, com opção de gravar ou abrir pela galeria.
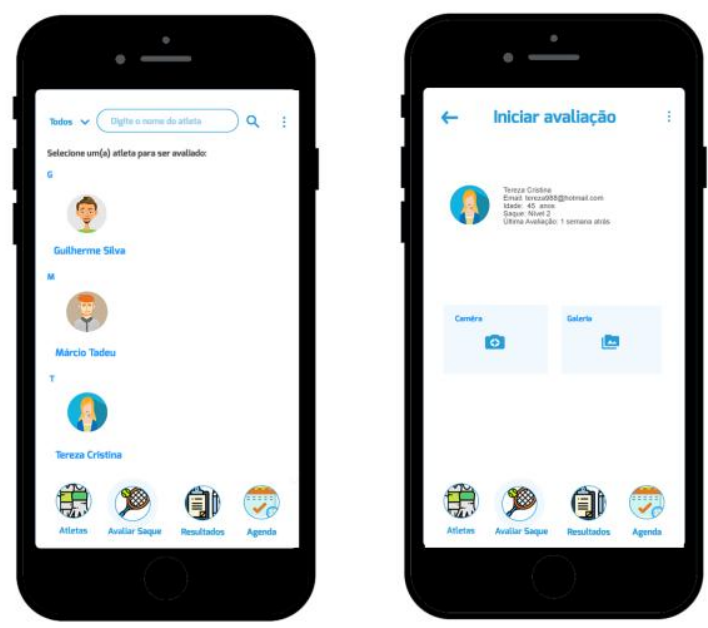

Figura 15 - (a) Seleção de atleta (b) Seleção de vídeo

A figura 16a apresenta a tela de cadastro de um novo atleta, onde o treinador deve preencher campos como: nome, email, idade, peso, altura, endereço e telefone. A figura $16 \mathrm{~b}$ apresenta a tela de configurações.
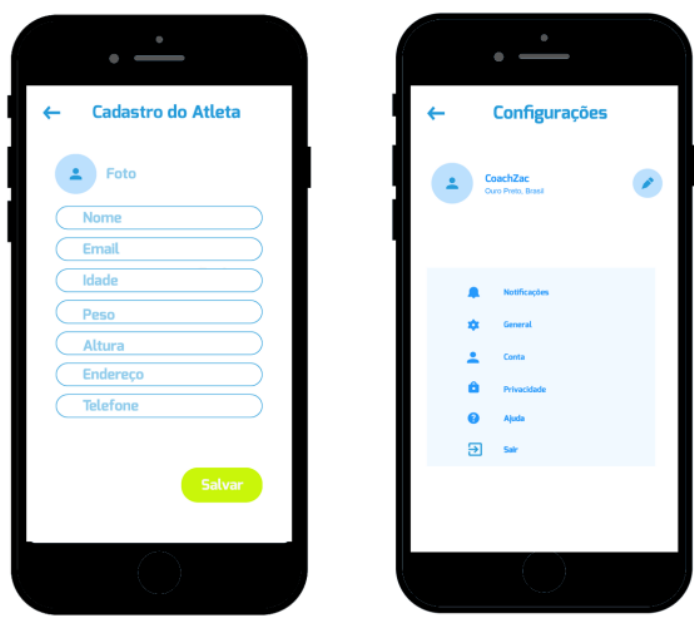

Figura 16 - (a) Cadastro do atleta (b) Configurações

A figura 17 apresenta a tela de avaliação do saque. No lado direito, estão representados todos os passos que devem ser avaliados pelo treinador, as funções de gravar áudios e criar notas de texto. Na parte inferior encontram-se as funções de reprodução de vídeo, como: avançar, voltar, câmera lenta e zoom.

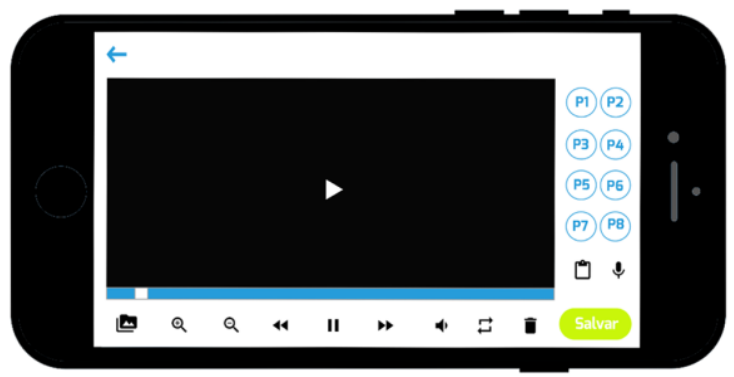

Figura 17 - Avaliação do saque

\section{Discussão dos Resultados}

Esta seção tem como objetivo interpretar os resultados com relação aos artefatos gerados durante o processo de design de interação. As reuniões com stakerholders e o resultado das 76 entrevistas, foram essências para gerar a lista de requisitos funcionais, reuisitos não-funcionais e personas. $\mathrm{O}$ modelo conceitual proposto permitiu a criação de metáforas de interação, com uma ampla identificação com o tênis, o que facilitou a compreensão das funcionalidades do CoachZac. As personas criadas permitiram o entendimento das características dos possíveis usuários finais do CoachZac, como se 
comportam e o que esperam da aplicação. Em especial, a persona da treinadora, que demonstrou frustração com os métodos convencionais de avaliação, e acredita que a tecnologia pode auxiliá-la. Através das personas, surgiram os atores dos cenários de interação do CoachZac. Os cenários foram uma boa forma de comunicação com os stakerholdes, porque são escritos em uma linguagem natural, como estórias. O diagrama de tarefas mostra a simplicidade do fluxo de tarefas do CoachZac. O modelo de interação identificou as decisões que deverão ser tomadas pelo usuário, para atingir seu objetivo final.

O modelo de dados contribui na percepção do mundo real em torno do problema e na definição de conjuntos de objetos básicos chamados entidades e nos relacionamentos entre esses objetos. O protótipo de baixa fidelidade gerou uma representação conceitual das necessidades dos usuários. O protótipo de alta fidelidade permitiu simular a interação dos usuários no CoachZac.

\section{Conclusões e Trabalhos Futuros}

Neste artigo foi apresentado o processo de design de interação de um aplicativo de mobile learning, CoachZac, para apoio a treinadores e atletas que almejam melhorar o fundamento do saque no jogo de tênis. O resultado atual é um protótipo de alta fidelidade, que permite simular a interação do usuário com o sistema, validando se suas necessidades foram atendidas. A princípio, o aplicativo contém o módulo do Treinador que permite que ele cadastre atletas, e avalie os saques, gerando resultados e feedbacks, com a possibilidade de compartilhar com os atletas.

Foi apresentado neste artigo um processo de design de interação com os diversos artefatos gerados em cada etapa: perfis de usuários, personas e objetivos associados, modelo de tarefas, modelo de interação, modelo de dados e protótipos de baixa e alta fidelidade. Espera-se assim chamar a atenção tanto da academia quanto da indústria para a importância das diversas representações de design ao longo do processo de construção de um software interativo. Esses artefatos, além de facilitarem a comunicação entre os membros da equipe de desenvolvimento, ajudam os próprios designers a refletirem sobre o que estão construindo e terem um maior entendimento das necessidades dos usuários em diversos níveis de abstração distintos.

As principais sugestões para trabalhos futuros incluem a realização de testes de interação com usuários finais do aplicativo, criação de um projeto visual mais refinado, contemplando nome, logotipo, slogan, projeto de cores, tipografia e criação dos ícones, desenvolvimento final do aplicativo para os sistemas operacionais Android e IOS. Um trabalho futuro de médio prazo inclui realizar o processo de design de interação e desenvolver o módulo do atleta, para que possam acompanhar seus resultados e interagir no aplicativo, e a utilização de tecnologias de processamento digital de imagens para automatizar as avaliações dos movimentos corporais do saque.

\section{Referências Bibliográficas}

[Barbosa. 2010] Barbosa, S. D. J; e da Silva, B. S. (2010). Interação humano-computador. Campus, 1 edição.

[EImasri, 2011] Elmasri, R.; Navathe, S. Sistemas de Banco de Dados, Pearson Addison Wesley, $6^{\mathbf{a}}$ ed., 2011.

[Fitivity, 2018] Fitivity. Tennis Training. Version 6.2.1. [S.1.: s.n.], 2018. Disponível em: <https://play.google.com/store/apps/details?id=com. fitivity.tennis_skills>. Acessoem: 21 jul. 2018.

[Hix, 1993] Hix, Deborah.; Rex Hartson, H.. Developing user interfaces: ensuring usability through product and process. John Wiley and Sons, 1993.

[Ishizaki, 2006] Ishizaki, Márcio T.; Castro, Mara. Tênis: Aprendizagem e Treinamento. 1.ed. São Paulo: Phorte, 2006.

[Marçal et al., 2009] Marçal, E.; Lima, L. D.; Júnior, M.; Viana, W.; Andrade, R.; Ribeiro, J. W. A Utilização de Dispositivos Móveis com Ambientes Tridimensionais como Ferramenta para Favorecer ao Ensino de Hardware. Anais do XIX Simpósio Brasileiro de Informática na Educação. 2009, Florianópolis. Disponível em: 


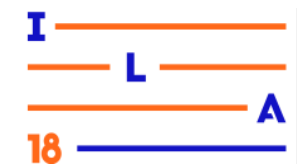

http://www.brie.org/pub/index.php/sbie/article/view/ 1146/1049>. Acesso em 17 jun. 2018.

[Mülbert, 2011] Mülbert, A. L.; Pereira, A. T. C. Um panorama da pesquisa sobre aprendizagem móvel (m-learning). Associação Brasileira de Pesquisadores em Cibercultura. 2011, Florianópolis. Anais do V Simpósio Nacional da ABCiber. Disponível em: <http://abciber.org.br/simposio2011/anais/Trabalhos /artigos/Eixo\%201/7.E1/80.pdf>. Acesso em 17 jun. 2018.

[Preece et al., 2007] Preece, J.; Rogers, Y.; Sharp, $H$. Interaction Design: beyond human-computer interaction. 2a Ed. England: John Wiley \& Sons, 2007.

[Rosson, 2002] Rosson, M.B. \& Carroll, J.M. Scenario-Based Development of HumanComputer Interaction. San Francisco, CA: Morgan Kaufmann Publishers, 2002.

[Soegaard, 2013] Soegaard, Mads.; Dam, Rikke Friis. The Encyclopedia of Human-Computer
Interaction, 2nd Ed. The Interaction Design Foundation, 2013. Disponível em:<https://www.interactiondesign.org/literature/bo ok/the-encyclopedia-of-human-computer-

interaction-2nd-ed/personas>. Acesso em: 19 out. 2018.

[Sommerville, 2011] Sommerville, Ian. Engenharia de Software, $9^{\text {a }}$ Edição. Pearson Education, 2011.

[Treuherz, 2006] Treuherz, Ralf Mário. Tênis: técnicas e táticas de jogo: preparação estratégica, mental, física, nutricional/ Treuherz \& Cornejo ; Prefácio Carlos Alberto Kirmayr ; Ilustrações Otoni Gali Rosa. - São Paulo : Alaúde Editorial, 2006.

[Zappasoft, 2018] Zappasoft. Tennis Coach Plus. Version 2.9. [S.1.:s.n.], 2018. Disponível em: $<$ https://play.google.com/store/apps/details?id=com. zappasoft.tennis\&hl=pt>. Acesso em: 21 jul. 2018. 